\title{
Origins of misorientation defects in single crystal castings: A time resolved in situ synchrotron $X$-ray radiography study
}

\author{
J.W. Aveson ${ }^{1,2, a}$, G. Reinhart ${ }^{3}$, H. Nguyen-Thi ${ }^{3}$, N. Mangelinck-Noël ${ }^{3}$, N. D’Souza ${ }^{4}$, and H.J. Stone ${ }^{1}$ \\ ${ }^{1}$ Department of Materials Science and Metallurgy, University of Cambridge, 27 Charles Babbage Road, Cambridge, \\ CB3 OFS, UK \\ ${ }^{2}$ Frazer-Nash Consultancy, Stonebridge House, Dorking Business Park, Dorking, RH4 1HJ, UK \\ ${ }^{3}$ IM2NP, UMR 7334 CNRS and Aix-Marseille University, Faculté des Sciences et Techniques, Avenue Escadrille \\ Normandie Niemen - Case 142, 13397 Marseille Cedex 20, France \\ ${ }^{4}$ Rolls-Royce plc. Derby, DE2,4 8BJ, UK
}

\begin{abstract}
The presence of grain boundaries in single crystal castings is intolerable owing to the detrimental impact on creep and fatigue behaviour. Whilst the origins of many defects such as freckles have been understood since the 1970s, other defects such as slivers, or indeed the small mosaicity observed in many castings have eluded comprehensive treatments. In the present work, in situ X-ray imaging has been used to examine the origin of misorientation defects that arise during solidification. Dendrite deformation was observed, which impacted growth characteristics and led to a permanent misoriention. Digital image correlation analysis showed the dendrites becoming increasingly bent as solidification progressed. In order to probe the deformation modes further, a method was devised to convert standard EBSD data into measurements of bending and torsion angle. It was demonstrated that such defects form as the result of bending moments arising from differential thermal contraction and gravity.
\end{abstract}

\section{Introduction}

Grain boundaries are axiomatically problematic in single crystal castings. Thus, there is a strong need to mitigate such defects through improvements in processing technology. In the casting process, there are three potent mechanisms by which misorientation defects can arise:

i nucleation of multiple grains

ii fragmentation of existing solid

iii unrecovered deformation of existing solid.

Thus, in order to tackle the problem of stray grains in a satisfactory manner, it is necessary to fully understand these three phenomena. From an experimental perspective, deformation during solidification is difficult to observe and quantify [1]. From a modelling and simulation approach, the change in geometry as a result of solidification needs to be incorporated into flow and structural solvers. Both of these present challenges that are not easily overcome.

Sliver defects occur regularly in directionally solidified single crystal castings (Fig. 1). They are elongated grains that extend along the solidification axis. Previous investigations showed that they are particularly prevalent at reductions in cross sectional area, and potentially linked to the presence of oxide residue [2]. This finding was corroborated by D'Souza and co-workers who were investigating novel mould geometries for seeded castings.

\footnotetext{
${ }^{\text {a }}$ Corresponding author: $\mathrm{j}$. aveson@fnc.co.uk
}

They found that sliver grains emanated from constricted channels that were designed to act as simplified grain selectors [3]. Investigations using finite element modelling and electron microscopy identified that the sliver defects formed at regions of high stress during solidification, and that the oxide defects found played no role [4].

Modelling and simulation, combined with ex situ micro structural characterisation have been used for many years to understand how solidification defects arise. However, in the last 15 years, radiographic investigation of solidification phenomena has become increasingly popular [5]. This allows for direct observation of microstructures as solidification progresses. In particular, it has been used to examine dendrite fragmentation in aluminium alloys, a phenomena only previously accessible by post mortem characterisation [1].

The purpose of the present study is to experimentally link in situ observation of deformation during solidification by radiography with electron backscattered diffraction mapping. This allows for the assessment of the underlying microstructural phenomena leading to the formation of macroscopic defects.

\section{Methods}

The aim of the present work was to experimentally investigate deformation of dendrites during solidification of the nickel-based superalloy CMSX-4. Two approaches were taken: solidification radiography was performed at

This is an Open Access article distributed under the terms of the Creative Commons Attribution License 4.0, which permits unrestricted use, distribution, and reproduction in any medium, provided the original work is properly cited. 


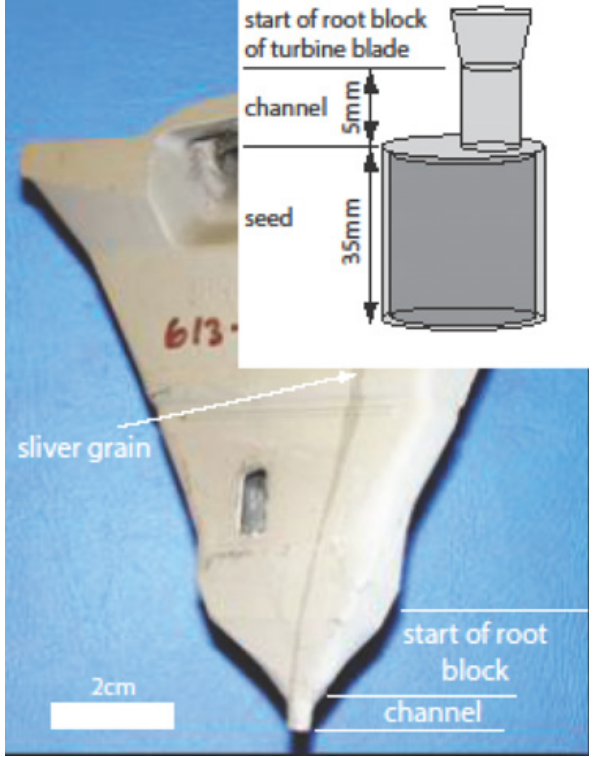

Figure 1. Photograph of a turbine blade with the sliver grain indicated. The inset shows a schematic of the mould configuration.

the European Synchrotron Radiation Facility to provide time resolved images of the solidification process and electron backscattered diffraction, to provide quantitative information about the dendrite misorientation across the casting.

Radiographic imaging was performed at the BM05 beam line of the European Synchrotron Radiation Facility. Thin sheet-like specimens $(40 \mathrm{~mm} \times 6 \mathrm{~mm} \times 0.3 \mathrm{~mm})$ were placed in BN coffins and sealed with Mo clips. These were mounted in a bespoke Bridgman furnace with long axis pointing vertically upward and the thin axis facing the beam, shown in Fig. 2. The furnace has been described in detail elsewhere [6]. Images were recorded with a frequency of $0.2 \mathrm{~Hz}$ using a Fast Readout Low Noise (FReLoN) CCD camera.

In order to improve contrast and provide background correction, pixel-wise image division was performed using a reference image of homogenous liquid. This was the only image processing applied to improve the quality of the images without leaving artefacts. The noise within the image was typically of the same length scale as the secondary dendrite arms, prohibiting smoothing in the Fourier domain.

Digital image correlation (DIC) was performed on the recorded images to provide information on the evolution of the strain field during solidification. The "Strainmaster" module within the DaVis software from LaVision $\mathrm{GmbH}$ was used for the analysis. This process uses standard optimisation methods to find the transformation that most closely maps one image onto another, by performing minimisation in the least squares sense. In the images shown, the rotation component of the displacement tensor is shown. The images acquired were treated using the sum of differential method, whereby the displacement vector field is calculated by summing the previous displacement vectors. When viewing dendrites, this has the effect of highlighting any bending within the plane of the image.

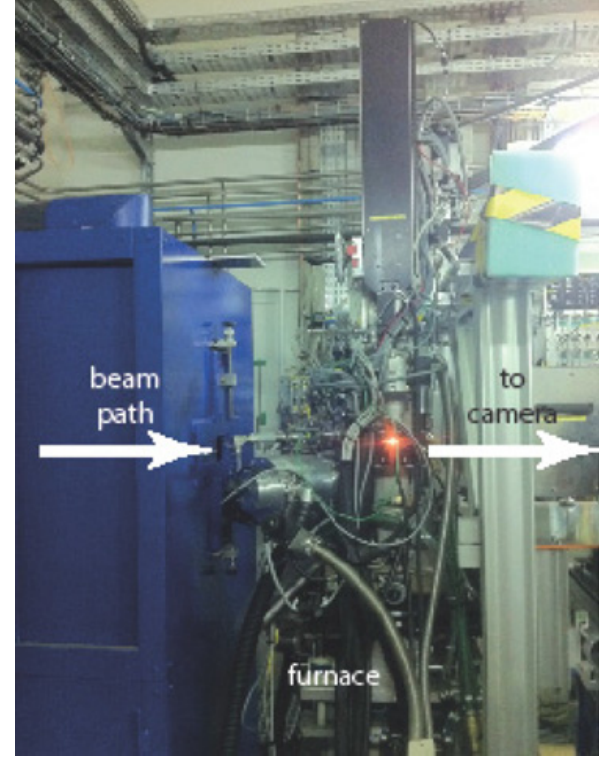

Figure 2. Photograph of the furnace used for the radiography studies.

Electron backscattered diffraction was performed on castings of the form shown in Fig. 1. Sections were taken through the axis of the casting in the channel. The metal was ground and polished using $\mathrm{SiC}$ paper, $3 \mu \mathrm{m}$ and $1 \mu \mathrm{m}$ diamond suspensions, and finally colloidal silica. The specimen was examined on a JEOL 840 scanning electron microscope with an Oxford Instruments hkl Channel 5 EBSD system.

Data analysis was performed using a bespoke routine coded in MATLAB. The steps in the calculation are shown in Fig. 3. The first stage is to convert the data to a quaternion description where the rotations are described by a unit vector with four parameters. This makes it straightforward to reduce the data from the 24 possible zones in cubic symmetry, down to a single zone. From here, the orientations can be averaged by taking the arithmetic mean of each of the parameters. This corresponds to the seed orientation. The angle between the primary axis of the seed and that at each pixel can be calculated using the scalar product. This corresponds to the angle through which a dendrite must have bent. By applying a rotation about the cross product of the primary axes of the seed and the measurement, such that they are brought to co-incidence, the secondary angle - the torsion angle - can be calculated. The detailed mathematics of the operation are given in Reference [4].

\section{Results}

Figure 4 shows the results of the DIC analysis performed on a sequence of radiographs taken during solidification. A pair of dendrites can be observed, growing from left to right. The colour overlay shows the magnitude of rotation about an axis coming out of the plane of the paper. In the colour version of the image, a rotation in an upwards sense is shown in red, and a rotation in a downwards sense is shown in blue. It can be seen that there is significant 


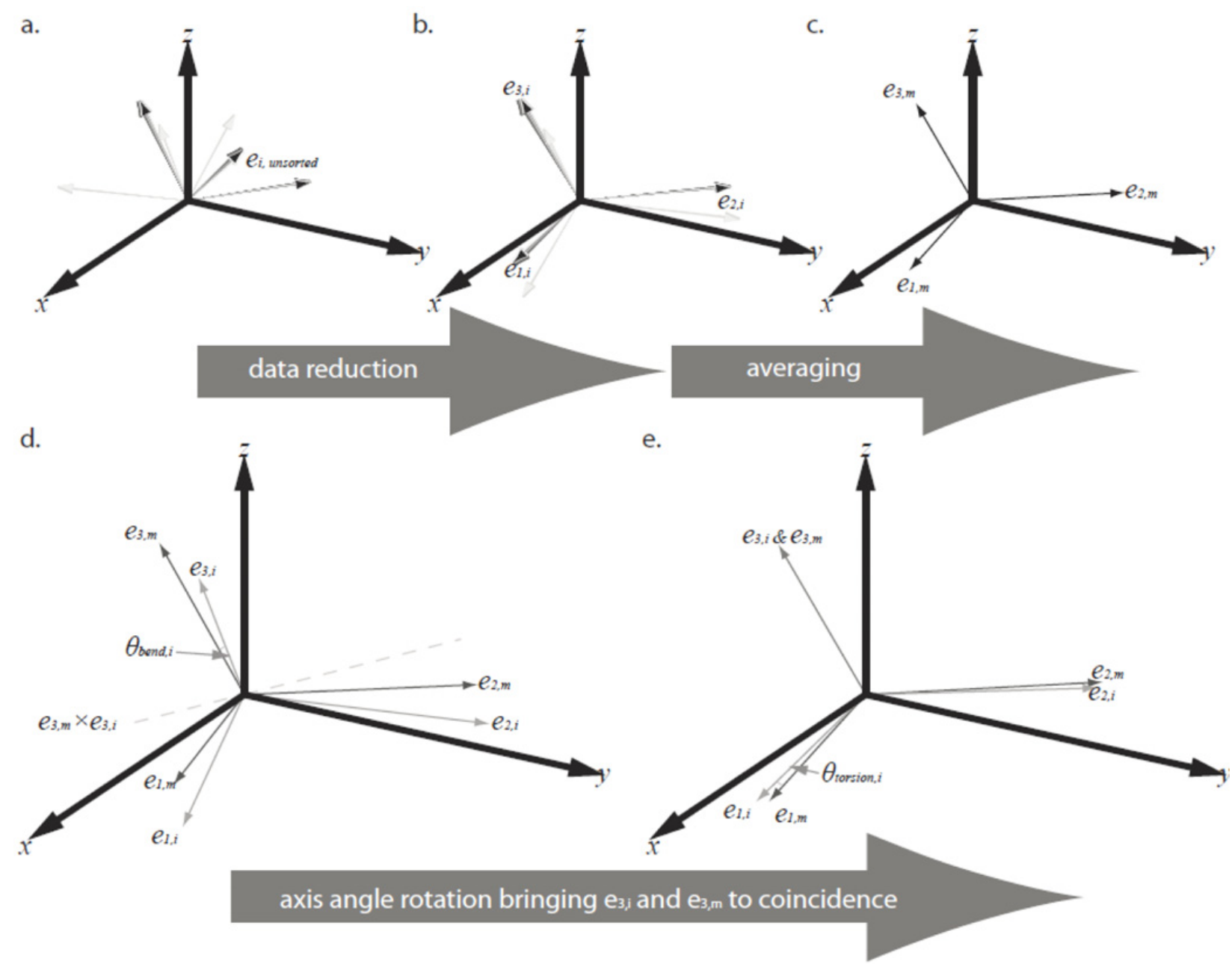

Figure 3. Sequence of illustrations showing the method used to perform the analysis of the EBSD data, as described in the text. This protocol has been used to produce the maps shown in Fig. 5.

distortion in on the right-hand side of the image. This is furthest away from the fixed point from which the dendrites have grown. The upper dendrite can be seen to have bent upwards as time elapsed, likewise the lower dendrite can be seen to have bent downwards as time elapsed. At additional positions along the length of the dendrite spine, there are several other concentrations of bending of smaller magnitude. Closer inspection shows that these are positions where there is appreciable bridging of secondary dendrite arms between the two primary dendrites.

The EBSD analysis gives a complementary picture of the deformation occurring during deformation. The two maps in Fig. 5 show the angle through which a dendrite has bent and twisted, as calculated using the method described in Section 2 and shown schematically in Fig. 3. In the orientation maps, the underlying dendrite microstructure can be clearly seen. This shows that the dendrites are of a different orientation to the surrounding interdendritic material. Examining the two maps, it can be seen that the extent of bending is significantly higher than that of torsion. Furthermore, the differing orientations are observed in clusters as highlighted in the map of bending.

\section{Discussion}

The combined picture from electron backscattered diffraction and X-ray radiography provides useful insight into the mechanics of dendrites during solidification. In two different specimen geometries, it can be seen that dendrites act as cantilever beams, and are anchored where they are surrounded by solid interdendritic material, as shown schematically in Fig. 6. This can be clearly seen in the DIC data, which shows misorientation accumulating along the length of primary dendrite arms. This is arguably the mechanism underlying the similar misorientation patterns measured by EBSD by Newell et al. [7,8]. Furthermore, it has recently been concluded elsewhere that sliver defects are a result of bending moments arising on dendrites from differential thermal contraction between mould and metal [4]. The direct observation of dendrite showing cantilever behaviour in the same alloy under similar processing conditions supports this conclusion.

Thus, when dendrites deform in the mushy zone, either elastically or plastically, as the interdendritic solidification front progresses, any residual deformation in the dendrite arm will be frozen in. This explains the result shown in Fig. 5, where dendrites have a different orientation to the interdendritic material. 


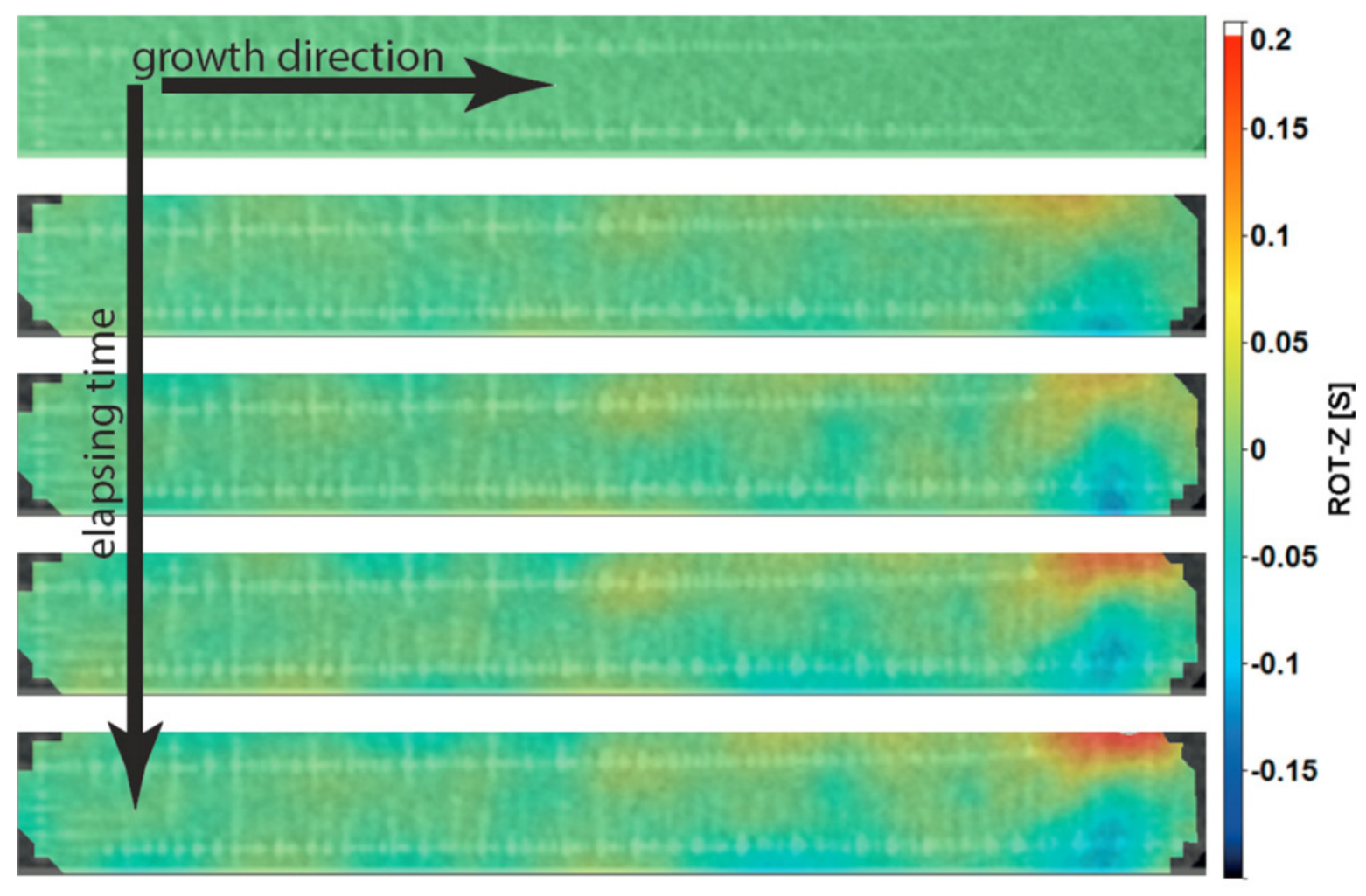

Figure 4. Radiographs with overlaid DIC maps showing rotation angles about an axis pointing out of the plane of the page.

\section{a. Bending}

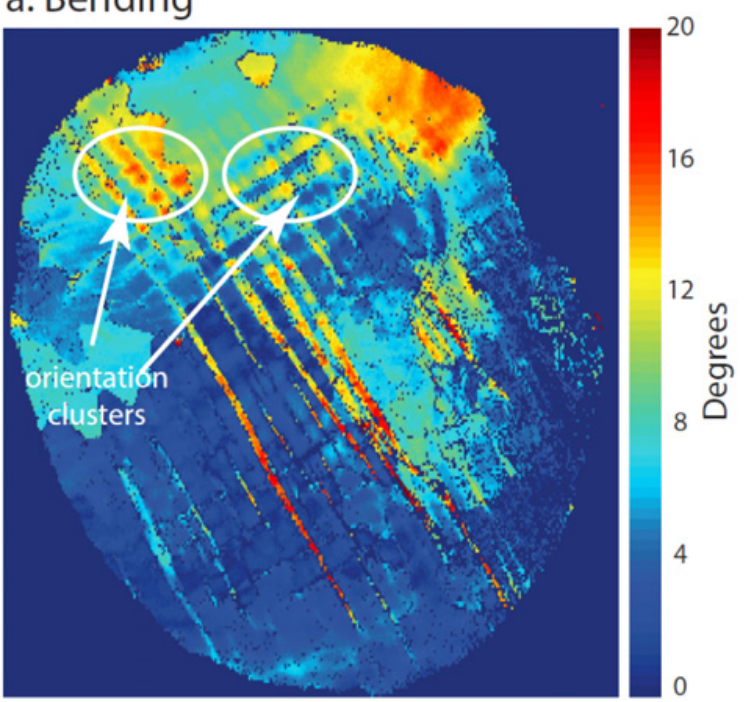

\section{b. Torsion}

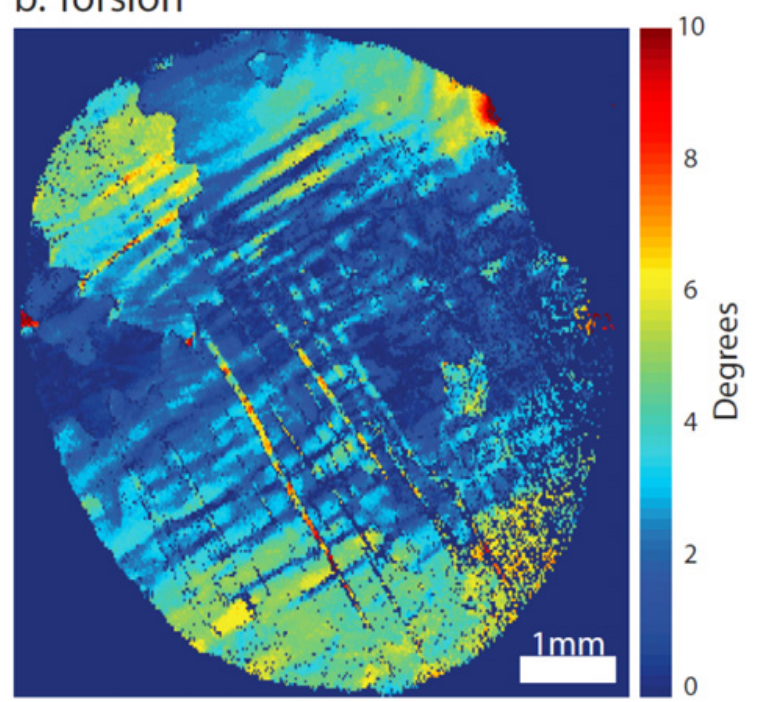

Figure 5. Maps showing dendrite: a. bending and b. torsion angles, as calculated by the method described in Section 2 and shown schematically in Fig. 3.

In addition, it was noted that patches of increased rotational activity could be seen where secondary dendrite arms from the neighbouring dendrites impinged on each other. Orientation clusters corresponding to neighbouring dendrites were also seen in the electron backscattered diffraction data. This shows that loads are readily transferred from dendrite to dendrite via impinged secondary arms. This leads to the collective motion of dendrites as a result of thermal loading, either from differential thermal contraction between mould and metal, or from self-stresses in the mushy state.
This pair of findings is likely to be of practical relevance in the casting of single crystal turbine blades. The in situ observation of solidification validates some of the assumptions and inferences made from EBSD and finite element simulations. These showed that whilst microstructural considerations such as the inclination of dendrites on the mould wall were important, sliver defects could be predicted as being more likely to occur where high stresses were experienced in the freshly solidified metal. Recent investigations indicated that extensive plastic deformation imparted 
a.
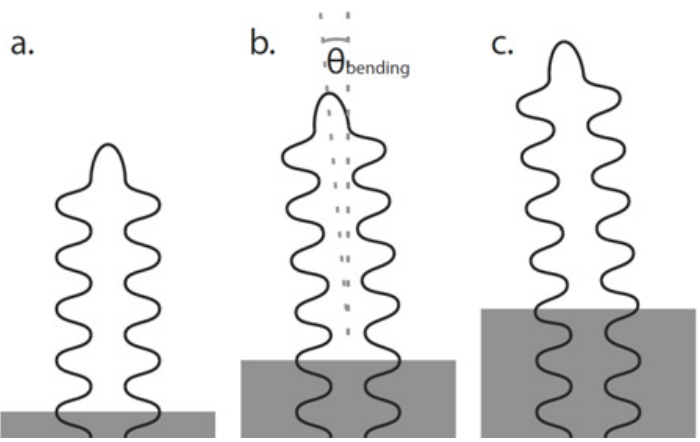

Figure 6. Schematic diagram showing how deformations can be locked in by an advancing interdendritic front.

to a casting at high temperatures can be responsible for subsequent recrystallisation during homogenisation heat treatments [9]. An additional boon of using casting simulation to avoid recrystallisation defects is that the same criteria will similarly evade sliver defects.

\section{Summary}

The use of synchrotron X-ray radiography, in combination with more traditional experimental tools, offers the possibility of distinguishing underlying mechanisms of defect formation at a conceptually finer scale. This study has found that the notion that dendrites deform as cantilever beams is correct. It has also shown load can be transferred between dendrites where secondary dendrites converge, leading to elevated deformation at these sites.
The authors are grateful to T.A. Lafford of the European Synchrotron Radiation Facility for assistance with the radiography portion of this study. B.J. Foss and B.A. Shollock are acknowledged for assistance with EBSD. J. R. Davenport and F. di Giocchino are acknowledged for assistance with DIC. The study was funded by the EPSRC/Rolls-Royce plc. Strategic Partnership under grants EP/H022309/1 and EP/H500375/1.

\section{References}

[1] G. Reinahrt, A. Buffet, H. Nguyen-Thi, B. Billia, H. Jung, N. Mangelinck-Noël, N. Bergeon, T. Schenk, J. Härtwig, J. Baruchel, Met. Mater. Trans. A39, 865 (2008)

[2] C.A. Carney and J. Beech, Proceedings of the 4th decennial conference on solidification processing. University of Sheffield; 1997. p 33

[3] X.L. Yang, P.D. Lee and N. D'Souza, JOM 57, 40 (2005)

[4] J.W. Aveson, P.A. Tennant, B.J. Foss, B.A. Shollock, H.J. Stone, N. D'Souza, Acta Mater, 61, 5162 (2013)

[5] H. Nguyen-Thi, L. Salvo, R.H. Mathiesen, L. Arnberg, B. Billia, M. Suery, G. Reinhart, Compes Rendus Physique, 327, 13 (2012)

[6] A. Tandjaoui, N. Mangelinck-Noël, G. Reinhart, J.-J. Furter, B. Billia, T. Lafford, J. Baruchel, X. Guichard, Energy Procedia, 27, 82 (2012)

[7] M. Newell, K. Devendra, P.A. Jennings, N. D’Souza, Mater. Sci. Eng. A412, 307 (2005)

[8] B. Billia, N. Bergeon, H. Nguyen-Thi, H. Jamgotchian, Phys. Rev. Lett. 93, 126105 (2004)

[9] C. Panwisawas, H. Mathur, J.-C. Gebelin, D. Putman, C.M.F. Rae, R.C. Reed, Acta Mater., 61, 51 (2013) 